\title{
Magnetic relaxation in chain-of-spheres ferromagnetic particles
}

\author{
Jyh-Shinn Yang ${ }^{\mathrm{a}, *}$, Ching-Ray Chang ${ }^{\mathrm{b}}$ \\ ${ }^{a}$ Institute of Optoelectronic Sciences, National Taiwan Ocean University, Keelung, Taiwan \\ ${ }^{\mathrm{b}}$ Department of Physics, National Taiwan University, Taipei, Taiwan
}

\begin{abstract}
The thermal activation of elongated ferromagnetic particles is analyzed using a chain-of-spheres model. The spheres within the chain are assumed to be coupled magnetically with dipolar interaction. The effect of uniaxial magnetocrystalline anisotropy along the chain is also taken into account. It was shown that the behavior of thermal switching critically depends on the relative strength of shape anisotropy and magnetocrystalline anisotropy, field orientation, sweep field rate and temperature. (C) 2002 Elsevier Science B.V. All rights reserved.
\end{abstract}

Keywords: Chain of spheres; Fanning; Magnetic relaxation

The requirement in miniaturization of the magnetic system enables the size of magnetic unit to remain shrinking, and then the size falls into the regime of single magnetic domain owing to the nanofabrication technology $[1,2]$. In sufficiently small particles, the exchange force maintains the magnetization uniform and the magnetization usually exhibits two stable equilibrium states separated by an energy barrier. Switching from one state to the other is achieved by a magnetic field or temperature. The thermal energy is comparable to the energy barrier, and the thermal fluctuation leads to a relaxation of the magnetization. The relaxation phenomenon is important and governs the long-term stability of magnetic system. From the viewpoints of both great technological importance and fundamental physics, a detailed understanding of the effect of thermally activated relaxation of the magnetization is warranted.

The rate of relaxation of magnetic system critically depends on the magnetization reversal mechanism, which cannot be described satisfactorily by coherent rotation mode. The chain-of-spheres is the first model to

\footnotetext{
*Corresponding author. Tel.: + 886-2-24622192; fax: + 8862-24634360.

E-mail address: jyang@mail.ntou.edu.tw (J.-S. Yang).
}

describe the incoherent magnetization reversal in elongated magnetic particles [3,4]. In recent years, the model has been shown to govern the magnetic reversal behavior of many magnetic systems, e.g. iron particles [5], Ba ferrite particles [6], and chains composed of nanosize ferromagnetic spheres [7], or nanowires [8]. For a basic understanding of incoherent magnetization reversal, the symmetric fanning mode is of great value, since it clearly provides a picture that at nucleation the equilibrium state of magnetization is such that the creation of additional magnetic poles should be largely avoided. Moreover, the results from fanning are very similar to those obtained by curling [4]. Up to now, the effect of thermal agitation for chain-of-spheres model is still not considered. In order to gain further insight into the magnetization reversal process of elongated particles, it becomes inevitable to study the thermal relaxation in the chain-of-spheres model.

The elongated ferromagnetic particle or nanowire is treated as a chain of $n$ equal spheres with each sphere assumed to be uniformly magnetized. The magnetostatic interaction among these spheres is taken into account, and additionally, a uniaxial magnetocrystalline anisotropy $K_{\mathrm{c}}$ along the chain axis is incorporated. Therefore, while the magnetic moment reverses via the symmetric fanning mode, the magnetic free energy of the chain in 
an applied field $\mathbf{H}$ is given by

$$
\begin{aligned}
E= & \pi M_{\mathrm{s}}^{2} \bar{K}_{n} V_{\mathrm{t}}\left[-\frac{1}{2}(\sin \beta \sin \theta \cos \phi+\cos \beta \cos \theta)^{2}\right. \\
& \left.+\frac{1}{4 f_{n}}\left(\sin ^{2} \theta \cos 2 \phi+\cos ^{2} \theta\right)-h \cos \theta\right]
\end{aligned}
$$

where $M_{\mathrm{S}}$ is the saturation magnetization, $V_{\mathrm{t}}$ is the volume of chain, $h=H / \pi M_{\mathrm{s}} \bar{K}_{n}, f_{n}=3 \bar{K}_{n} / 2 L_{n}, \bar{K}_{n}=$ $K_{n}+K_{\mathrm{c}} /\left(\pi M_{\mathrm{s}}^{2} / 2\right)$, and the coefficients $K_{n}$ and $L_{n}$ are defined as in Refs. [2,3], $\beta$ is the angle between the chain axis and the field, $\theta$ the polar angle between the sphere moment and the field, and $\phi$ the azimuthal angle of sphere moments, measured from the plane made by the chain and the field. It is noted that the sign of $\phi$ alternates in sign from one sphere to the next along the chain, but $|\phi|$ remains constant.

In order to treat the effect of thermal relaxation, the chain is treated as having two levels, which are occupied with probabilities $P_{1}$ and $P_{2}$, respectively. Its evolution is given by the master equation [9]

$\mathrm{d} P_{1} / \mathrm{d} t=-\kappa_{12} P_{1}+\kappa_{21} P_{2}=-\mathrm{d} P_{2} / \mathrm{d} t$,

where $\kappa_{i j}=\kappa_{i \rightarrow j}=f_{0} \exp \left(-Q_{i} / k_{\mathrm{B}} T\right)$ is the transition rate from level $i$ to $j$ level. For the frequency prefactor, we choose the value $f_{0}=e^{25} \mathrm{~Hz}, k_{\mathrm{B}}$ is the Boltzmann constant, $T$ is the temperature, and $Q_{i}$ is the energy barrier to be overcome on departure from the level $i$. In order to drive the hysteresis loop, the applied field is assumed to vary with time in a sinusoidal way, $H(t)=H_{0} \cos (2 \pi f t)$, where $f$ is sweep rate and the amplitude $H_{0}$ is large enough to saturate the magnetization.

The dependence of coercivity on field orientation is shown in Fig. 1 and exhibits a salient feature of fanning mode for low magnetocrystalline anisotropy. The coercivity initially increases slowly by increasing the

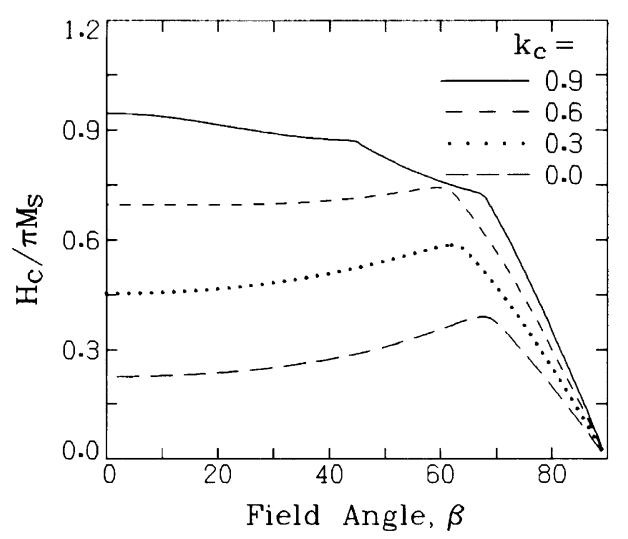

Fig. 1. The effect of magnetocrystalline anisotropy on coercivity of fanning mode for chain-of-spheres particles. Here, $n=$ $50, f=100 \mathrm{~Hz}, \pi M_{\mathrm{s}}^{2} \bar{K}_{n} / 2 k_{\mathrm{B}} T=100$, and $k_{\mathrm{c}}=2 K_{\mathrm{c}} / \pi M_{\mathrm{s}}^{2}$. field angle and reaches a peak at some certain field angle, and then decreases rapidly to zero till the field angle is $90^{\circ}$. As expected, the chain with larger magnetocrystalline anisotropy has a higher energy barrier to slow down the thermal relaxation, and results in the higher coercivity. Moreover, the peak of coercivity shifts towards small field orientation and the regime for fanning mode decreases as the magnetocrystalline anisotropy increases. In comparison with the experimental data [7], the results obtained by fanning are in qualitative agreement with the observed behavior for the dependence of coercivity on the field angle.

The number of spheres in the chain or the aspect ratio of particle also influences the relaxation rate. The coercivity initially increases rapidly with the number of spheres and then approaches a finite value for a large number of spheres (Fig. 2). This is due to the higher aspect ratio leading to the larger shape anisotropy. However, the shape anisotropy has an upper bound, since $K_{n}=1.202$ and $L_{n}=1.052$ as $n \rightarrow \infty$. In comparison with coherent rotation mode, the coercivity of fanning mode is found to lower significantly at small and intermediate field angles, suggesting that the fanning mode indeed provides a fast thermal relaxation channel for the chain-of-spheres particles. The influence of temperature on the coercivity is shown in Fig. 3. As the temperature is elevated, the transition rate increases and the magnetization more easily jumps over the barrier, resulting in the lower coercivity. It is noted that the coercivity does not depend on the temperature for a large field angle, since the reversal mechanism follows the coherent rotation mode as the field angle is in this regime [5]. The influence of field sweep rate on the thermal switching is also studied. It is shown that the coercivity increases with increasing sweep rate, resulting from the short waiting time of measurement.

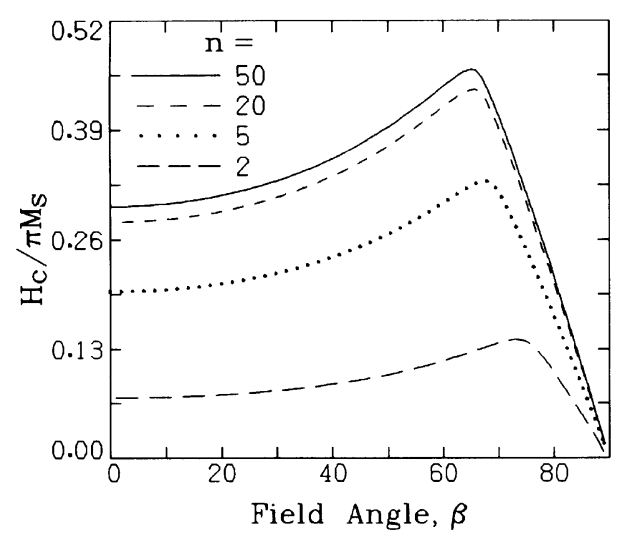

Fig. 2. The effect of aspect ratio on coercivity of fanning mode for chain-of-spheres particles. Here, $f=100 \mathrm{~Hz}$, $\pi M_{\mathrm{s}}^{2} \bar{K}_{n} / 2 k_{\mathrm{B}} T=100$, and $2 K_{\mathrm{c}} / \pi M_{\mathrm{s}}^{2}=0.1$. 


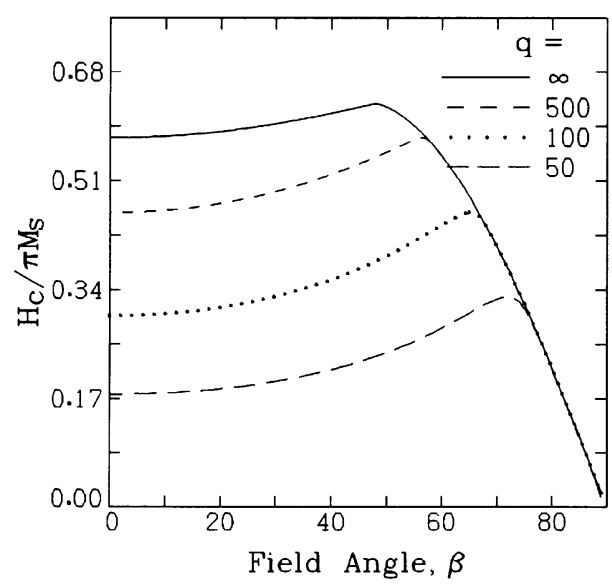

Fig. 3. The effect of temperature on coercivity of fanning mode for chain-of-spheres particles. Here, $n=50, f=100 \mathrm{~Hz}, k_{\mathrm{c}}=$ $2 K_{\mathrm{c}} / \pi M_{\mathrm{s}}^{2}=0.1$, and $q=\pi M_{\mathrm{s}}^{2} \bar{K}_{n} / 2 k_{\mathrm{B}} T$.

In conclusion, we have incorporated the thermal relaxation into the chain-of-spheres model. It is shown that the thermal magnetic switching follows the fanning mode for small magnetocrystalline anisotropy at a small field orientation. However, large magnetocrystalline anisotropy can modify the thermal switching to exhibit coherent-rotation-like behavior. For a large field orientation, the thermal magnetic switching always shows coherent-rotation-like behavior.

This work was supported by the National Science Council of ROC under Grant No. NSC89-2112-M-019008 .

\section{References}

[1] S.Y. Chou, Proc. IEEE 85 (1997) 652.

[2] R.P. Cowburn, J. Phys. D: 33 (2000) R1.

[3] I.S. Jacobs, C.P. Bean, Phys. Rev. 100 (1955) 1060.

[4] H.J. Richter, J. Magn. Magn. Mater. 154 (1996) 263.

[5] Y. Luo, J.-G. Zhu, IEEE Trans. Magn. 30 (1994) 4080.

[6] D.H. Han, H.L. Luo, Z. Yang, J. Appl. Phys. 78 (1995) 1146.

[7] L. Zhang, A. Manthiram, Phys. Rev. B 54 (1996) 3462.

[8] Y. Peng, H.-L. Zhang, S.-L. Pan, H.-L. Li, J. Appl. Phys. 87 (2000) 7405.

[9] J.-S. Yang, C.-R. Chang, I. Klik, Phys. Rev. B 51 (1995) 15203. 